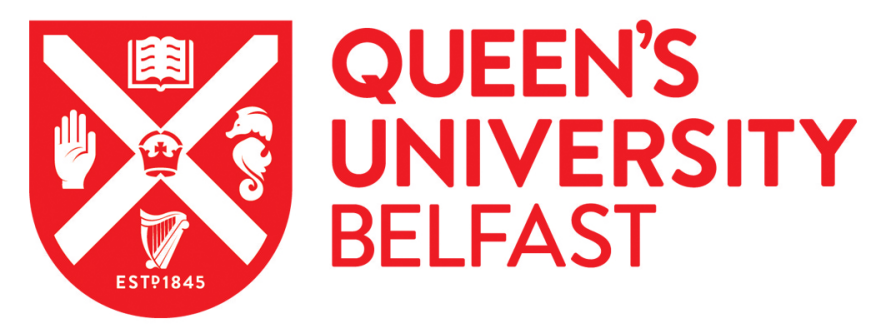

\title{
Pilot tone reference-less phase conjugator for phase modulated retrodirective antenna applications
}

Buchanan, N., \& Fusco, V. (2016). Pilot tone reference-less phase conjugator for phase modulated retrodirective antenna applications. IEEE Antennas and Wireless Propagation Letters, 15, 298-300.

https://doi.org/10.1109/LAWP.2015.2442296

Published in:

IEEE Antennas and Wireless Propagation Letters

Document Version:

Peer reviewed version

Queen's University Belfast - Research Portal:

Link to publication record in Queen's University Belfast Research Portal

Publisher rights

( 2015 IEEE. Personal use of this material is permitted. Permission from IEEE must be obtained for all other uses, in any current or future media, including reprinting/republishing this material for advertising or promotional purposes, creating new collective works, for resale or redistribution to servers or lists, or reuse of any copyrighted component of this work in other works.

\section{General rights}

Copyright for the publications made accessible via the Queen's University Belfast Research Portal is retained by the author(s) and / or other copyright owners and it is a condition of accessing these publications that users recognise and abide by the legal requirements associated with these rights.

Take down policy

The Research Portal is Queen's institutional repository that provides access to Queen's research output. Every effort has been made to ensure that content in the Research Portal does not infringe any person's rights, or applicable UK laws. If you discover content in the Research Portal that you believe breaches copyright or violates any law, please contact openaccess@qub.ac.uk. 


\title{
Pilot tone reference-less phase conjugator for phase modulated retrodirective antenna applications
}

\author{
Neil Buchanan, Vincent Fusco, Fellow, IEEE
}

\begin{abstract}
This paper presents a simple tracking PLL which can be used to track phase modulated signals and provide a phase conjugated signal for retrodirective retransmission. The configuration allows the retrodirective antenna to directly track phase modulated signals with no requirement for a separate $\mathrm{CW}$ pilot tone. The ability to directly track phase modulated signals is carried out using a $4 \mathrm{X}$ multiplier on the tracking PLL reference signal. Practical phase conjugation results are presented for a 5 element retrodirective array simultaneously sending and receiving phase modulated (QPSK) signals. Signals with levels as low as $\mathbf{- 1 2 2}$ $\mathrm{dBm}$ can be phase conjugated and retransmitted with $30 \mathrm{dBm}$ EIRP.
\end{abstract}

Index Terms-Phased arrays, Phase modulation, Phase locked loops, Phase conjugation

\section{INTRODUCTION}

$\mathrm{R}$ ETRODIRECTIVE antennas have many promising applications, for example, in satellite communications [1]. Satellite applications place a lot of challenges on the retrodirective antenna, namely weak received signal and complex modulation schemes. Recent retrodirective antennas [2] use a phase tracking PLL to track the phase of a weak received signal in the presence of a strong retransmitted signal. These systems are capable only of tracking CW signals, or modulation schemes such as amplitude shift keying, where there is no phase variation. QPSK signals can be tracked using a Costas loop [3], or a multiply, filter, divide architecture [4]. However these architectures are difficult to realise with discrete components and operation at low signal to noise ratios can be problematical.

In this paper we describe a tracking PLL architecture capable of tracking QPSK signals, as well as more complex schemes up to 256 QAM. The proposed phase locked loop is no more complicated, in terms of component count, than a basic CW tracking PLL and is capable of simultaneously phase conjugating received signals as low as $-122 \mathrm{dBm}$, in the presence of retransmitting a signal with an EIRP of up to $30 \mathrm{dBm}$.

\section{Phase Modulation Tracking Phased LOCKED loOP}

The phase modulation Tracking Phased Locked Loop (PLL) shown in Fig. 1 is an adaption of a classical phaselock receiver [5]. The main change proposed to the phaselock receiver is making the reference signal presented to the phase detector 4 times the frequency of the intermediate frequency signal $\left(\mathrm{F}_{\mathrm{IF}}\right)$ it is being locked to. The operation of the PLL is explained with reference to the timing diagram in Fig. 2. Here the reference signal, and a QPSK signal are shown, as square waves, assuming a limiting operation within the EXOR phase detector. Referring to Fig. 2(a), the reference signal is shown as a square wave of $4 \mathrm{X}$ the frequency of the IF signal. Scenario (i) shows the output of the phase detector (Exclusive OR operation), with no phase lag applied to the IF signal. This phase detector output is passed through a loop filter to produce an average DC signal, shown as the dotted line Fig. 2(a). Scenario (ii) in Fig. 2(a) shows the effect when the IF signal has a phase lag, this is equivalent to angle of arrival changing in an antenna array. When the phase lag is present an additional pulse starts to appear which raises the average DC level of the phase detector output. This would have the effect, in the PLL of Fig. 1, to momentarily shift the frequency of the VCO until the IF is again locked in phase to the reference signal. It is important that the IF square wave has a slightly shorter "on" state than "off" state. This is necessary to avoid a second pulse appearing on the falling edge of the IF signal, since this would cancel the effect of the pulse generated from the leading edge. A slightly shorter "on" time than "off" time is produced by the phase detector IF input [6] threshold levels. The IF input recognises an "on" state as $>0.2 \mathrm{~V}$ and anything below this as an "off” state. If the phase detector is being driven by a sine wave signal of $1 \mathrm{~V} \mathrm{p}$-p, or $\pm 0.5 \mathrm{~V}$, the phase detector internal IF waveform will transition the $\pm 0.5 \mathrm{~V}$ input signal to the "on" state at $0.2 \mathrm{~V}$ resulting in a square wave with an unequal duty cycle with shorter "on" state compared to "off" state.

Fig. 2(b) shows the actual waveforms obtained from the experimental setup of Fig. 1. Note that reference and IF waveforms appear sinusoidal since limiting action is internal to the phase detector and cannot be directly measured. From Fig.

Belfast, Northern Ireland Science Park, Queen’s Road, Queen’s Island, Belfast, United Kingdom, BT3 9DT, Tel +44 2890 971806, Fax +44 2890971702 (v.fusco@ecit.qub.ac.uk).
Manuscript received March 12th, 2015.

Vincent Fusco and Neil .B. Buchanan are with the Institute of Electronics, Communications and Information Technology (ECIT), Queen's University 
2 , if the IF phase was to be shifted by $0^{\circ}, 90^{\circ}, 180^{\circ}$ or $270^{\circ}$ then the IF signal would always have the same relationship to the reference signal, since one cycle of the reference signal is equivalent to a $90^{\circ}$ shift in the IF waveform. This has the result that the DC output of the phase detector is unchanged with the four $90^{\circ}$ states of QPSK modulation.

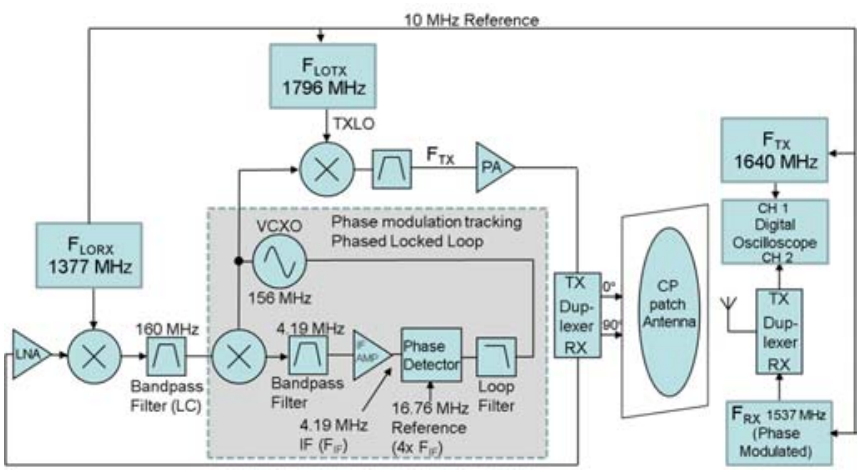

Fig. 1. Phase Locked Loop for phase modulation tracking

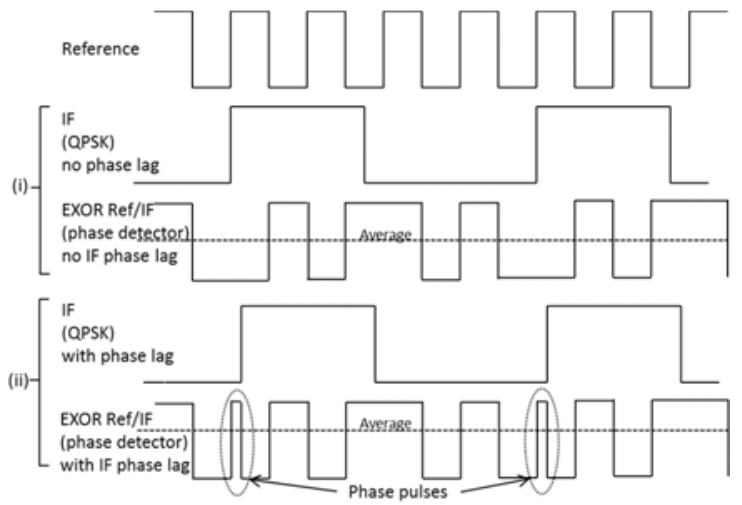

(a) Timing diagram

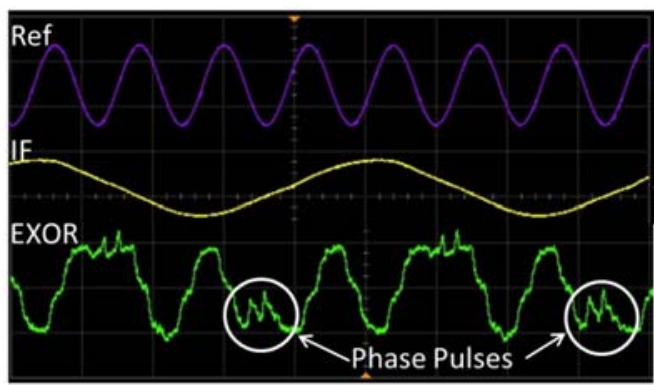

(b) Measured phase detector waveforms

Fig. 2. Phase Detector Waveforms

\section{EXPERIMENTAL RESULTS}

\section{A. Phase conjugating results}

A phase conjugating cell has been designed (Fig. 1) and employs a double superheterodyne receiver/transmitter arrangement to allow it to operate with the high level of specification required for satellite communication systems with regards to image rejection, selectivity, and so on. The module operates at $\mathrm{L}$ band with a receive frequency in the $1.5 \mathrm{GHz}$ range, and transmit frequency in the $1.6 \mathrm{GHz}$ range. The PLL loop filter design is critical to obtain low phase noise and jitter on the output signal [7]. To provide stable performance to low signal levels, a $2^{\text {nd }}$ order active loop filter was used with a cutoff frequency of $100 \mathrm{~Hz}$. Another critical component for low signal level operation in the receiver chain is the LNA, which has a gain of $>20 \mathrm{~dB}$ with a noise figure of $<2 \mathrm{~dB}$. The mixers used were active types, typically having conversion gains in the region of $10 \mathrm{~dB}$ and noise figures of around $5 \mathrm{~dB}$.

A phase modulated transmit signal of $20 \mathrm{kbps}$ is chosen to provide an optimum match to the module's IF filters. A 1377.73 $\mathrm{MHz}$ local oscillator is used for the receiver section, which down converts to a first IF of $160.19 \mathrm{MHz}$. This is then further down converted to a second IF of $4.19 \mathrm{MHz}$ by mixing with a $156 \mathrm{MHz} \mathrm{VCXO}$, which forms part of the phase tracking circuit. The $156 \mathrm{MHz}$ VCXO tracks the phase of the received signal, and is up converted by mixing with a TX LO signal of $1796 \mathrm{MHz}$. This up converting action of $\mathrm{F}_{\mathrm{LO}}-\mathrm{F}_{\mathrm{VCXO}}=\mathrm{F}_{\mathrm{TX}}$, provides the phase conjugation for the retransmit signal.

The resultant IF signal time domain waveforms, taken from the inputs to the phase detector (Fig. 1), allow all the four phase states of the QPSK signal to be captured is shown in Fig. 3. Each of the four QPSK phase states bears the same phase relationship to the reference signal, thus providing a stable average output of the phase detector, after low pass filtering.

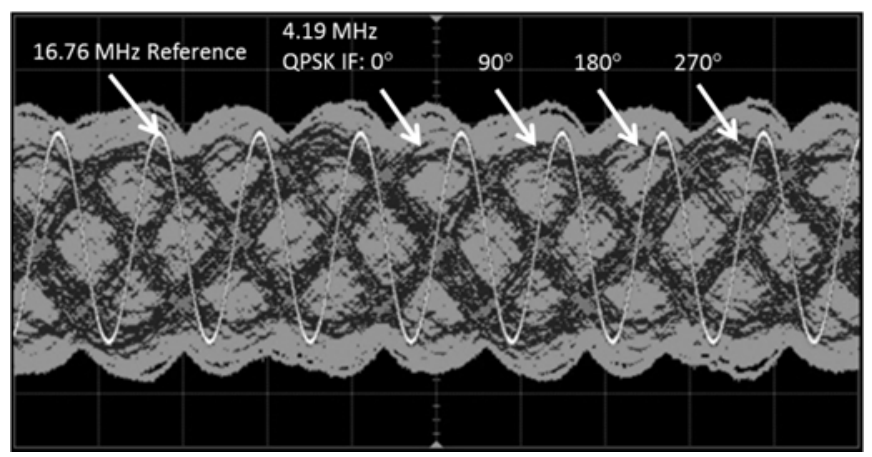

Fig. 3. Reference and QPSK IF time domain waveforms

The phase conjugating cell was measured down to low received signal levels using the setup of Fig. 1 . The received signal level at the antenna was determined accurately from the IF signal amplitude output of the $4.19 \mathrm{MHz}$ IF amplifier, since the conversion gain, from the RX port to IF output, of the phase conjugating cell had been previously measured. Accurate phase conjugation of QPSK to $-122 \mathrm{dBm}, 16 \mathrm{QAM}$ to $-121 \mathrm{dBm}$, and 256 QAM down to $-119 \mathrm{dBm}$ was obtained (Fig 4). The retransmitted EIRP of a single antenna module with $6.5 \mathrm{dBi} \mathrm{CP}$ antenna is about $30 \mathrm{dBm}$ making the arrangement presented here viable for applications such as Inmarsat BGAN [1] where the received signal strengths of the spot beams are in the region of $-110 \mathrm{dBm}$ at the antenna, and retransmit EIRP in the region of $38 \mathrm{dBm}$, i.e. 6 modules each with a retransmit EIRP of $30 \mathrm{dBm}$. 


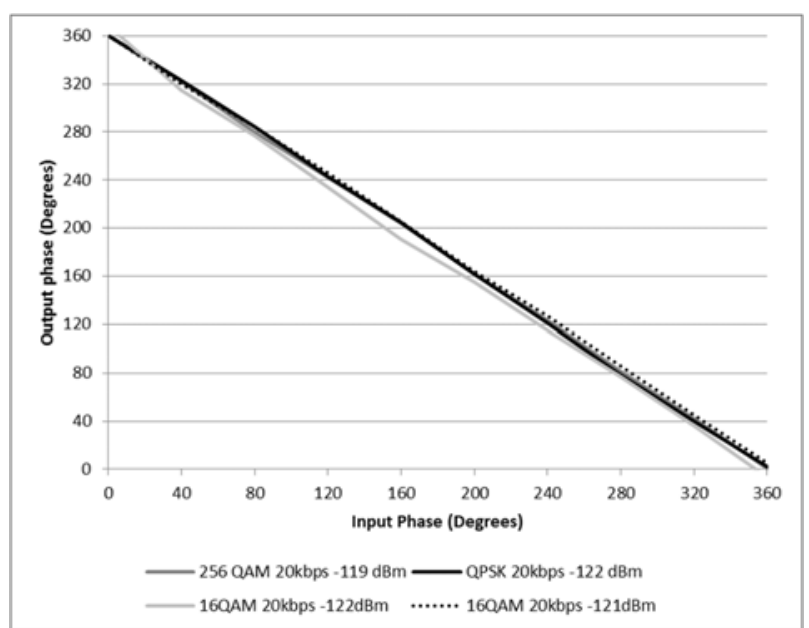

Fig. 4. Reference and QPSK IF time domain waveforms

\section{B. Retrodirective measurements - Transmit Mode}

The antenna element setup of Fig. 1 was configured as a $5 \times 1$ element retrodirective array, operating in full QPSK duplex transmit/receive mode and its monostatic radiation patterns measured by applying a QPSK received signal to the array and measuring the QPSK modulated retransmitted signal. A QPSK modulated signal was applied to the TXLO port (Fig. 1). The transmitted radiation pattern was measured with regard to the retransmitted Error Vector Magnitude (EVM), rather than retransmitted amplitude, Fig. 5, since EVM is a more suitable benchmark for retransmitted modulated signal quality, ensuring that all transmitted symbols are correctly aligned across the array. Measured single element EVM is approximately 2\% higher than that of the 5 element array. With all five elements switched on a flat $5 \%$ EVM versus azimuth response is obtained over the azimuth range $\pm 40^{\circ}$, a $5 \%$ EVM equates to a BER of less than $10^{-6}$ [8] for QPSK.

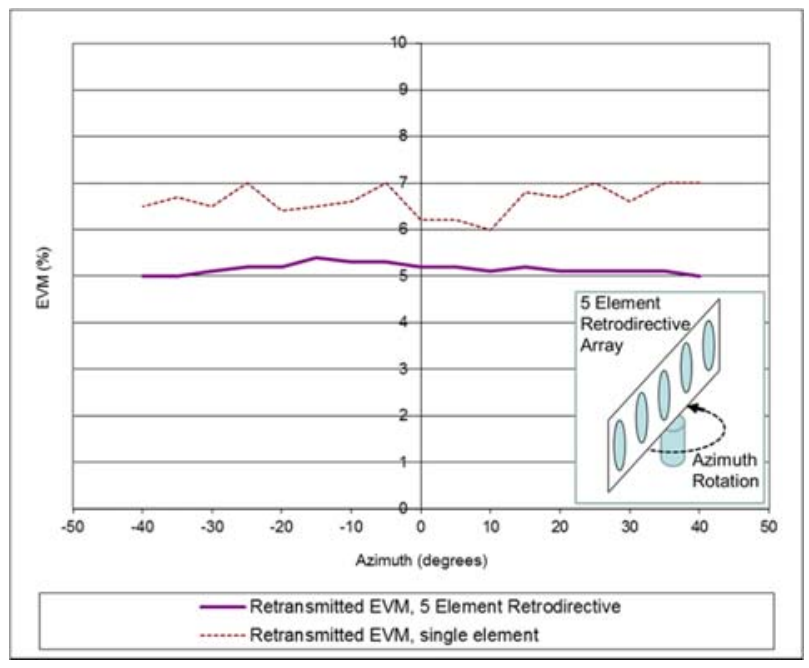

Fig. 5. Retransmitted EVM Vs azimuth for 5 element retrodirective array

\section{Retrodirective measurements - Receive Mode}

The retrodirective array was shown practically to have the ability to optimally combine the received signal. The method used involves combining a constant phase IF signal from each element, such that the IFs always combine in phase regardless of angle of arrival. This mode of operation follows the principles of [9], with the exception that, in this case, phase modulated signals can be used instead of CW signals. A fairly flat EVM response for the array was achieved on receive mode, varying from $6.2 \%$ to $7.2 \%$ over for an azimuth range of $-40^{\circ}$ to $40^{\circ}$, confirming optimal phase combination on receive mode over a wide azimuth range.

\section{CONCLUSIONS}

The retrodirective antenna has been shown to directly track QPSK/16QAM/256QAM signals of levels as low as $-122 \mathrm{dBm}$ whilst being able to simultaneously transmit/receive digital modulation. The tracking PLL configuration used within the retrodirective antenna is no more complex than a conventional phaselock receiver requiring only the addition of a $4 \mathrm{X}$ multiplier on the reference signal.

\section{REFERENCES}

[1] Inmarsat BGAN, http://www.inmarsat.com/service/bgan/, Accessed on 3-7-2014

[2] Buchanan, N., Fusco, V., Van Der Vorst, M., Williams, N., Winter, C., "New Retrodirective Antenna Techniques for Mobile Terminal Applications” 32nd Antenna Workshop, ESA/ESTEC, Noordwijk, The Netherlands, 5-8 October 2010

[3] Costas, J.P. "Introduction to Synchronous Communications", Proceedings of the IEEE, December 1956

[4] Steber, J.M. "PSK Demodulation: Part 1", WJ Tech Notes 1984, Vol. 11 No. 2 March/April 1984

[5] Gardner, F. M., Phaselock Techniques, John Wiley \& Sons Inc., New York, NY, 1979, p145

[6] Philips Semiconductor, “74HC4046 Phase-locked-loop with VCO”, Data Sheet, , Nov 251997

[7] P. V. Brennan, Phase-locked loops: principles and practice, London: Macmillan Press Ltd., 1996. pp 76-89.

[8] Shafik R.A., Rahman M.S., Islam, A.R., Ashraf, N. S., “On the Error Vector Magnitude as a performance Metric and Comparitive Analysis,” ICET 2006, NWFPUET, Peshawar

[9] Buchanan, N., Fusco, V., Van Der Vorst, M., “A High Performance Analogue Retrodirective Phase Conjugation Circuit with RX Array Factor Combination Ability” IMS 2011, Baltimore 